(C) 2017 IEEE. Personal use of this material is permitted. Permission from IEEE must be obtained for all other uses, in any current or future media, including reprinting/republishing this material for advertising or promotional purposes, creating new collective works, for resale or redistribution to servers or lists, or reuse of any copyrighted component of this work in other works. 


\title{
Synthesis of Unequally Spaced Linear Antenna Arrays with Minimum Element Spacing Constraint by Alternating Convex Optimization
}

\author{
Pengfei You, Yanhui Liu, Member, IEEE, Shu-Lin Chen, Kai Da Xu, Member, IEEE, Weiwen Li, and \\ Qing Huo Liu, Fellow, IEEE
}

\begin{abstract}
A novel method called alternating convex optimization is presented to synthesize unequally spaced linear arrays with minimum element spacing constraint. In this method, the problem of synthesizing an unequally spaced array is formulated as a sequence of alternating convex optimization problems, and the excitation vector and auxiliary weighting vector are alternately chosen as the optimization variables. The minimum spacing constraint for considering the physical element antenna size can be easily imposed in this alternating optimization process. Two examples for synthesizing unequally spaced linear arrays with focused and shaped patterns are provided to validate the effectiveness and advantages of the proposed method.
\end{abstract}

Index Terms-Unequally spaced linear array, alternating convex optimization, radiation pattern synthesis, minimum spacing constraint.

\section{INTRODUCTION}

$\mathbf{U}$ NEQUALLY spaced antenna arrays have been widely used in sonar, satellite communication and radar systems, due to the advantages such as suppressing the sidelobe and grating lobe levels as well as reducing the number of element required for desired pattern characteristics [1], [2]. As is well known, synthesizing an unequally spaced array with optimized element positions is a highly nonlinear optimization problem. The stochastic optimization algorithms such as genetic algorithm (GA) [3], [4], differential evolution algorithm (DEA) [5], [6] and particle swarm optimization (PSO) method [7], [8], would be appropriate since they can potentially find the globally optimal solution even with some complicated constraints such as power pattern shape control and minimum element spacing constraint. However, these methods are usually time-consuming especially when both the positions and complex excitations for a large number of elements need to be optimized. Some other synthesis techniques including the compressive sensing (CS) based synthesis approaches in [9], [10], Bayesian CS based synthesis approaches in [11]-[13],

Manuscript received xxx. This work was supported by the Science and Technology Research Project of Fujian Province under Grant No 2017I0017, the National Natural Science Foundation of China (NSFC) under Grant No. 61301009, and the Fundamental Research Funds for the Central Universities under Grant No. 20720160081. (Corresponding author: Yanhui Liu).

P. You, Y. Liu, K. D. Xu and W. Li are with the College of Electronic Science and Technology and Institute of Electromagnetics and Acoustics, Xiamen University, Fujian 361005, China (email: yanhuiliu@xmu.edu.cn).

$\mathrm{S}$. Chen is with the Global Big Data Technologies Centre, University of Technology Sydney (UTS), NSW 2007, Australia.

Q. H. Liu is with the Department of Electrical and Computer Engineering, Duke University, Durham, NC 27708, USA. the matrix pencil methods in [14], [15] and the reweighted $\ell_{1}$-norm optimization methods in [16]-[18], are much more efficient than the stochastic optimization algorithms. However, these interspacing-unconstrained methods usually are not able to control the minimum element spacing, and sometimes the synthesized arrays cannot be realized in practice.

Recently, some merging techniques have been developed in which synthesis results from CS or the reweighted $\ell_{1}$-norm optimization methods are double-checked, and then the closelyspaced elements are merged to meet the prescribed minimum element spacing constraint [19]-[22]. The merging techniques improve the availability of interspacing-unconstrained methods by utilizing a post-processing to control the minimum element spacing. However, the extra merger will result in unrecoverable pattern deterioration. In this work, we present a novel synthesis method which formulates the problem of synthesizing an unequally spaced array as a sequence of alternating weighted $\ell_{1}$-norm optimizations. In this method, the excitation vector and weighting vector are alternately chosen as the optimization variables. The minimum spacing constraint can be easily imposed in the alternating optimization process. Consequently, the minimum element spacing can be controllable and the presence of electrically small element spacings is prevented in the synthesis result. Since each iteration can be solved by convex optimization, the proposed method is called the alternating convex optimization. Two unequally spaced linear array synthesis examples are provided: one is for synthesizing a focused beam and the other is for a shaped pattern, both with prescribed minimum element spacings. The synthesis results show the effectiveness and advantages of the proposed method.

\section{FORMUlation AND Algorithm}

\section{A. Problem Description}

The synthesis of unequally spaced linear array can be formulated as finding the best element positions and excitations from a predefined closely spaced linear array. Let us consider a linear array with $N$ initial element positions which are assumed to be closely distributed along the $Z$-axis, with the spacing of $d \ll \lambda$. The array factor can be written as

$$
f(\theta)=\mathbf{a}(\theta)^{T} \mathbf{w}
$$

where

$$
\begin{gathered}
\mathbf{w}=\left[w_{1}, w_{2}, \ldots, w_{N}\right]^{T} \\
\mathbf{a}(\theta)=\left[e^{j \beta d \cos \theta}, e^{j 2 \beta d \cos \theta}, \ldots, e^{j N \beta d \cos \theta}\right]
\end{gathered}
$$


In the above, $j=\sqrt{-1}, \beta=2 \pi / \lambda$ is the wavenumber in free space, and the superscript ' $T$ ' denotes the transpose of a matrix.

Now, the problem is to find no more than $K(K<N)$ element positions from the $N$ initial positions with appropriate excitations, under multiple constraints such as pattern performance requirement and the minimum spacing constraint. Mathematically, that is,

$$
\begin{aligned}
& \text { find } \mathbf{w} \\
& \text { s.t. } \begin{cases}\|\mathbf{w}\|_{0} \leq K \\
d_{\min } \geq Q d \\
(F . B . C)\left\{\begin{array}{l}
f\left(\theta_{0}\right)=1 \\
|f(\theta)| \leq U_{\mathrm{SL}}(\theta),
\end{array}\right. \\
o r \\
(S . B . C) \begin{cases}\frac{\left|f(\theta)-f_{d}(\theta)\right|}{\left|f f_{d}(\theta)\right|} \leq \epsilon, & \forall \theta \in \Omega_{S L} \\
|f(\theta)| \leq U_{\mathrm{SL}}(\theta), & \forall \theta \in \Omega_{S L}\end{cases} \end{cases}
\end{aligned}
$$

where $\|\mathbf{w}\|_{0}$ denotes the number of non-zero components of $\mathbf{w}$ (i.e., the number of selected elements), $d_{\min }$ is the minimum spacing between the selected elements and $Q$ is a positive integer. The constraints (F.B.C) and (S.B.C) are used to control the radiation characteristics on the focused beam and the shaped pattern, respectively. $\theta_{0}$ denotes the look direction, $U_{\mathrm{SL}}(\theta)$ is a given upper bound over the sidelobe region $\Omega_{S L}$, $f_{d}(\theta)$ is the desired field pattern in the mainlobe region $\Omega_{M L}$ , and $\epsilon$ is the degree of accuracy.

\section{B. Alternating Convex Optimization (ACO) Synthesis}

1) Basic ACO synthesis: Obviously, the above problem is non-convex due to the $\ell_{0}$-norm constraint as well as the minimum spacing constraint. As is well known, the reweighted $\ell_{1}$-norm optimization is a convex problem but leads to a sparse solution. This technique has been successfully used to synthesize both the focused beam and shaped pattern problems in [16]-[18]. However, the disadvantage with the reweighted $\ell_{1}$-norm optimization is that the minimum spacing constraint cannot be easily incorporated in this technique. To overcome this problem, we will develop an alternating convex optimization-based synthesis method in which the minimum spacing constraint can be easily incorporated into the synthesis procedure.

At first, we consider the following optimization problem

$$
\begin{gathered}
\min _{\mathbf{w} \in \mathbb{C}^{N}, \mathbf{g} \in \mathbb{R}^{N}} \mathbf{g}^{T}|\mathbf{w}| \\
\text { s.t. }\left\{\begin{array}{l}
(F . B . C) \text { or }(S . B . C) \\
\mathbf{0} \preceq \mathbf{g} \preceq \mathbf{1} \\
\mathbf{1}^{T} \mathbf{g}=N-K
\end{array}\right.
\end{gathered}
$$

where $|\mathbf{w}|$ represents the $N$-dimensional excitation amplitude vector, and $\mathbf{g}$ is a weighting vector. Since both $|\mathbf{w}|$ and $\mathbf{g}$ are optimization variables, thus the above problem is called the biconvex optimization problem [23]. Obviously, for a fixed g, the objective function is convex with respect to the variable $\mathbf{w}$, and vice versa. Hence, one practical method is applying an alternating convex optimization strategy to solve (5) [23]. That is to solve a sequence of alternating weighted $\ell_{1}$-norm optimization problems:

$$
\begin{cases}\min _{\mathbf{w} \in \mathbb{C}^{N}} & \mathbf{g}_{*}^{T}|\mathbf{w}| \\
\text { s.t. } & (F . B . C) \text { or }(S . B . C) \\
\min _{\mathbf{g} \in \mathbb{R}^{N}} & \mathbf{g}^{T}\left|\mathbf{w}_{*}\right| \\
\text { s.t. } \quad\left\{\begin{array}{l}
\mathbf{0} \preceq \mathbf{g} \preceq \mathbf{1} \\
\mathbf{1}^{T} \mathbf{g}=N-K
\end{array}\right.\end{cases}
$$

In Problem (6a), $\mathbf{w}$ is the optimization variable under a given $\mathbf{g}_{*}$ that is obtained by solving Problem (6b) in the previous step, and in Problem (6b), $\mathrm{g}$ is the optimization variable under a given $\mathbf{w}_{*}$, the solution to Problem (6a). In the first iteration, $\mathbf{g}_{*}$ should be initialized. For example, we can choose $\mathbf{g}_{*}=1$. Clearly, both Problem (6a) and (6b) are weighted $\ell_{1}$-norm minimization problems. They can be solved by convex optimization. The whole procedure can be called the alternating convex optimization (ACO) method. In this method, the weighting vector $\mathbf{g}_{*}$ can be dynamically updated by successively solving the constrained optimization problem of (6b). In this way, the minimum spacing constraint can be easily incorporated in the whole iteration process by appropriately modifying the problem of $(6 b)$, which will be described in the next subsection. This is much different from the conventional reweighted $\ell_{1}$-norm method in which the weighting factor is usually simply set as the inverse of the amplitude of excitation plus a small positive number [16]-[18], and the minimum spacing constraint is hard to be dealt with.

2) ACO with minimum spacing constraint: These are obvious in the ACO method: g obtained from Problem (6b) will have ' 1 ' for $N-K$ entries and ' 0 ' for others, provided that there are no two identical excitation amplitudes in $\mathbf{w}_{*}$. In each iteration, the entries of ' 1 ' in $\mathbf{g}_{*}$ will maximally penalize the corresponding excitation amplitudes in $|\mathbf{w}|$ for Problem (6a), and the excitations corresponding to the ' 0 ' entries of $\mathbf{g}_{*}$ will be retained. In this situation, we can control the distribution of ' 1 ' entries in the $\mathbf{g}_{*}$ to determine which elements will be discarded.

Now, we consider how to add a constraint to control the minimum spacing between the selected elements. Assume that there exists an excitation distribution which has the minimum spacing of $Q d$. That is, there are at least $(Q-1)$ unselected elements between any two neighboring selected elements. In particular, for any of $Q$-length segment of $\mathbf{w}_{*}$, we have at most one selected element and at least $(Q-1)$ unselected ones. For Problem (6b), such an excitation distribution requires $\mathbf{g}$ satisfying the following constraint:

$$
\begin{aligned}
& \mathbf{1}^{T} \mathbf{g}(m: m+Q-1) \geq Q-1 \\
& \text { (for } m=1, \ldots, N-Q+1)
\end{aligned}
$$

This constraint can be easily incorporated into Problem (6b), and the resulting whole ACO method can control the minimum spacing constraint on the synthesized excitations.

\section{The Proposed ACO Synthesis Procedure}

The proposed ACO procedure for synthesizing unequally spaced linear arrays with minimum spacing constraint is listed in Algorithm 1. 


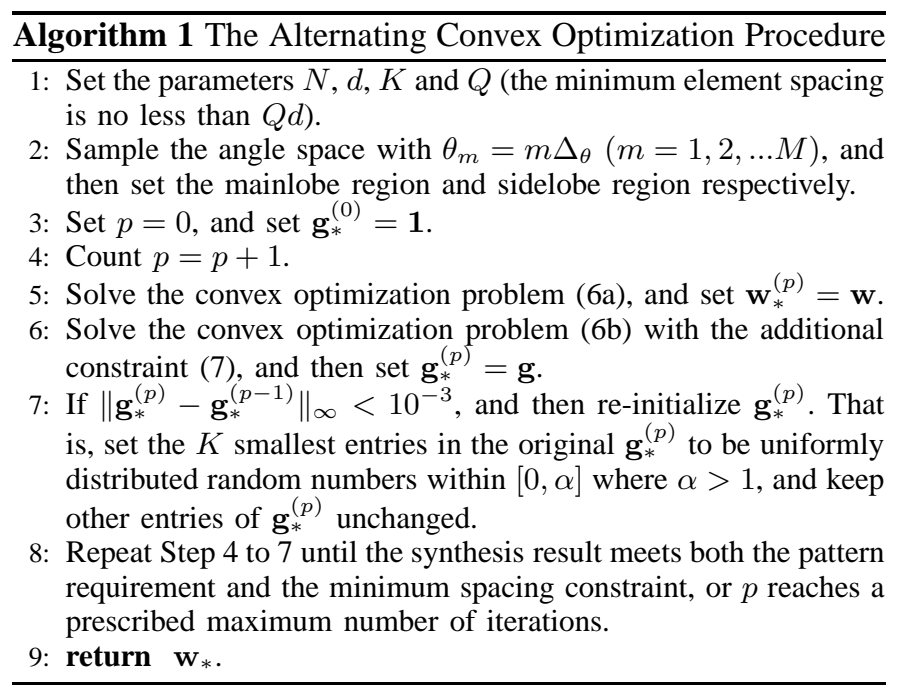

\section{NUMERICAL EXAMPLE}

\section{A. Scannable Focused Beam Synthesis}

In the first example, we consider a full-space scannable focused beam pattern which has the maximum sidelobe level (SLL) of $-30.3 \mathrm{~dB}$ for $0.125 \leq \cos \theta-\cos \theta_{0} \leq 2$ and $-21.3 \mathrm{~dB}$ for $-2 \leq \cos \theta-\cos \theta_{0} \leq-0.125$. Such a pattern was synthesized in [18] by using sequential convex optimization (the reweighted $\ell_{1}$-norm optimization), as shown in Fig. 1(a). The obtained array has 22 unequally spaced elements occupying an aperture of $D=9.66 \lambda$. The minimum spacing obtained in [18] is $0.34 \lambda$. In [2], a similar 22 -element unequally spaced array with the same minimum spacing is obtained by using the fast iterative soft-thresholding algorithm. For the proposed ACO method, we set $N=967$, $d=0.01 \lambda$, and $K=22$. Set $Q=35,36, \ldots, 46$ for checking the robustness of the proposed method with different minimum spacings (i.e., $0.35 \lambda, 0.36 \lambda, \ldots, 0.46 \lambda$, respectively). With these constraints, the proposed method can always give a satisfied pattern meeting the prescribed SLL bounds except for the case of $Q=46$ where the minimum spacing of $0.46 \lambda$ is actually the average spacing $(D /(K-1)=0.46 \lambda)$. For further comparison, we also apply the merging technique presented in [20] where some closely-spaced elements obtained from CS synthesis are merged and then the obtained element positions are successively perturbed to compensate for the pattern deterioration caused by the merging operation. The same set of minimum spacings are used. However, the merging technique can give a satisfied pattern result only when the minimum spacing is no larger than $0.42 \lambda$. For a larger minimum spacing requirement, this technique fails due to unrecoverable pattern deterioration in the merging process. Fig. 1(a) shows the synthesized patterns by all these methods and the proposed method (among all, the proposed method

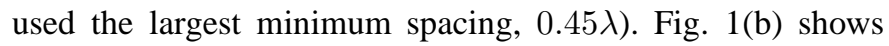
the beam pattern dependence with respect to the scan angle $\left(\cos \theta_{0}=-1,-0.9, \ldots, 1\right)$ of the synthesized array at the largest minimum spacing obtained by the proposed method. It is more intuitive to show the ability of the synthesized result to scan the full-space. Table I shows the synthesized excitations

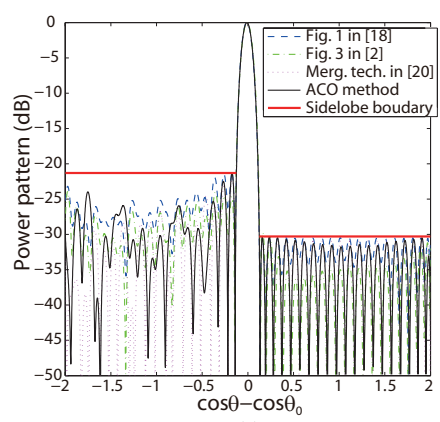

(a)

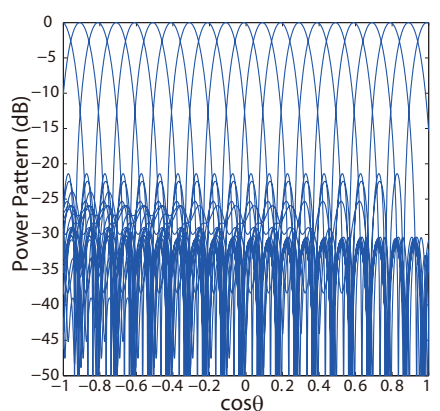

(b)
Fig. 1. (a) The full-space scannable focused beam patterns synthesized by the sequential convex optimization (SCO) in [18], the fast iterative softthresholding algorithm (FISTA) in [2], the merging technique in [20] and the proposed ACO method with $Q=45$. The obtained arrays all have 22 unequally spaced elements occupying an aperture of $9.66 \lambda$, and the obtained minimum spacing is $0.34 \lambda, 0.34 \lambda, 0.42 \lambda$ and $0.45 \lambda$, respectively. (b) The beam pattern dependence with respect to the scan angle of the synthesized array obtained by the proposed ACO method with $Q=45$.

TABLE I

THE SYNTHESIZED ELEMENT POSITIONS AND EXCITATIONS BY THE PROPOSED ACO METHOD FOR THE ARRAY PATTERN SHOWN IN FIG. 1

\begin{tabular}{c|c|c|c}
\hline $\mathrm{i}$ & Position $(\lambda)$ & Excit. Ampl. & Excit. Phase $\left(^{\circ}\right)$ \\
\hline 1 & 0 & 0.3292 & -28.19 \\
\hline 2 & 0.45 & 0.3209 & -23.35 \\
\hline 3 & 0.90 & 0.4208 & -17.32 \\
\hline 4 & 1.35 & 0.5311 & -11.29 \\
\hline 5 & 1.80 & 0.6162 & -5.07 \\
\hline 6 & 2.27 & 0.6976 & -6.21 \\
\hline 7 & 2.73 & 0.7784 & -3.39 \\
\hline 8 & 3.20 & 0.8455 & -4.13 \\
\hline 9 & 3.67 & 0.9229 & -4.65 \\
\hline 10 & 4.13 & 0.9735 & -1.39 \\
\hline 11 & 4.60 & 1 & -1.66 \\
\hline 12 & 5.06 & 1 & 1.66 \\
\hline 13 & 5.53 & 0.9735 & 1.39 \\
\hline 14 & 5.99 & 0.9229 & 4.65 \\
\hline 15 & 6.46 & 0.8455 & 4.13 \\
\hline 16 & 6.93 & 0.7784 & 3.39 \\
\hline 17 & 7.39 & 0.6976 & 6.21 \\
\hline 18 & 7.86 & 0.6162 & 5.07 \\
\hline 19 & 8.31 & 0.5311 & 11.29 \\
\hline 20 & 8.76 & 0.4208 & 17.32 \\
\hline 21 & 9.21 & 0.3209 & 23.35 \\
\hline 22 & 9.66 & 0.3292 & 28.19 \\
\hline & & &
\end{tabular}

and the selected element positions for the proposed method with $Q=45$.

\section{B. Flat-top Pattern Synthesis}

In this example, we consider a shaped pattern that has a flattop mainlobe with $40^{\circ}$ beamwidth and $\pm 0.22275 \mathrm{~dB}$ response ripple, as shown in Fig. 2(a). Its sidelobe level is less than $-30 \mathrm{~dB}$ in the two side regions of $\left[0^{\circ}, 65^{\circ}\right]$ and $\left[115^{\circ}, 180^{\circ}\right]$. This pattern was synthesized in [24] by the semi-definite programming (SDP) with a prefixed element positions where the minimum spacing is only $0.25 \lambda$. In [16], the reweighted $\ell_{1}$-norm optimization with a conjugate-symmetric excitation assumption was applied to reduce the number of elements from this array, and 31 elements were finally selected. The minimum spacing is not shown in [16]. Here, we apply the method in [16] to reproduce the synthesis result, and the obtained minimum spacing remains $0.25 \lambda$. By taking the 


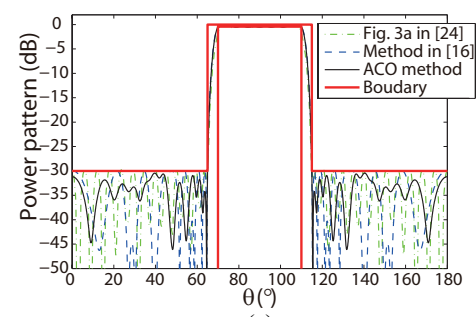

(a)

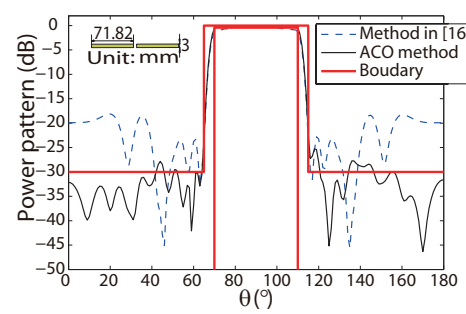

(b)
Fig. 2. (a) The flat-top patterns synthesized by the semi-definite programming (SDP) in [24] with a prefixed 41 element positions, the method in [16] with 31 elements, and the proposed ACO method with only 17 elements, respectively. The obtained minimum spacing is $0.25 \lambda, 0.25 \lambda$ and $0.64 \lambda$, respectively. (b) The real array patterns including mutual coupling by using HFSS simulation for the dipole array using the element excitations and positions synthesized by the method in [16] and the proposed ACO method.

same mainlobe pattern obtained by the method in [16] as the reference, we can apply the proposed ACO method to produce a new sparse array. A minimum constraint of $0.6 \lambda$ ( $Q=60$ and $d=0.01 \lambda$ ) is added. The obtained array has only 17 elements with the realized minimum spacing equal to $0.64 \lambda$ (the obtained minimum spacing may be larger than the prescribed criterion according to the constraint of (7)). Fig. 2(a) shows the comparison of the patterns obtained by [24], [16] and the proposed method. Further, we also give a comparison to check the real pattern of the dipole array using the element excitations and positions obtained by the method in [16] and the dipole array pattern using the synthesis results obtained by the proposed ACO method, respectively. The dipole works at the frequency of $1 \mathrm{GHz}$. The real array patterns are simulated by using High Frequency Structure Simulator (HFSS) software. The comparison results are plotted in Fig. 2(b). It is seen that although the real patterns for the both dipole arrays have increased sidelobe levels due to mutual coupling, the dipole array synthesized by the method in [16] with $0.25 \lambda$ minimum spacing has nearly $11.9 \mathrm{~dB}$ SLL degradation while the dipole array obtained by the proposed ACO method with $0.64 \lambda$ minimum spacing has about $4.7 \mathrm{~dB}$ SLL degradation. Table II shows the synthesized excitations and the selected element positions by the proposed method.

\section{CONCLUSION}

In this paper, a new method called alternating convex optimization has been proposed to synthesize the unequally spaced array with focused or shaped pattern. In this method, we express the unequally spaced array synthesis problem as performing a sequence of two alternating convex problems. The minimum spacing constraint can be easily incorporated in the array synthesis process rather than used as post-processing in some element-merging techniques. This can overcome the possible performance degradation in the element-merging operation. Synthesis results for two unequally spaced array with focused and shaped patterns show that the proposed method can obtain satisfactory pattern results with the controllable minimum element spacings, avoiding the presence of small minimum element spacings in synthesis results obtained by some interspacing-unconstrained methods. This would be very
TABLE II

THE SYNTHESIZED ELEMENT POSITIONS AND EXCITATIONS BY THE PROPOSED ACO METHOD FOR THE ARRAY PATTERN SHOWN IN FIG. 2

\begin{tabular}{c|c|c|c}
\hline $\mathrm{i}$ & Position $(\lambda)$ & Excit. Ampl. & Excit. Phase $\left({ }^{\circ}\right)$ \\
\hline 1 & 0 & 0.0224 & 180 \\
\hline 2 & 1.30 & 0.0233 & 0 \\
\hline 3 & 2.55 & 0.0325 & 180 \\
\hline 4 & 3.79 & 0.0482 & 0 \\
\hline 5 & 5.14 & 0.0759 & 180 \\
\hline 6 & 6.47 & 0.1219 & 0 \\
\hline 7 & 7.81 & 0.2198 & 180 \\
\hline 8 & 9.09 & 0.6594 & 0 \\
\hline 9 & 9.74 & 1 & 0 \\
\hline 10 & 10.38 & 0.6357 & 0 \\
\hline 11 & 11.68 & 0.2177 & 180 \\
\hline 12 & 12.99 & 0.1263 & 0 \\
\hline 13 & 14.32 & 0.0822 & 180 \\
\hline 14 & 15.62 & 0.0539 & 0 \\
\hline 15 & 16.91 & 0.0342 & 180 \\
\hline 16 & 18.15 & 0.0239 & 0 \\
\hline 17 & 19.47 & 0.0229 & 180 \\
\hline
\end{tabular}

essential for practical antenna array design. It should be noted that the proposed method can be extended to synthesize the planar and conformal unequally spaced arrays with the minimum spacing constraint.

\section{REFERENCES}

[1] V. Murino, A. Trucco, and C. S. Regazzoni, "Synthesis of unequally spaced arrays by simulated annealing," IEEE Trans. Signal Process., vol. 44, no. 1, pp. 119-122, Jan. 1996.

[2] X. Wang, E. Aboutanios, and M. G. Amin, "Thinned array beampattern synthesis by iterative soft-thresholding-based optimization algorithms," IEEE Trans. Antennas Propag., vol. 62, no. 12, pp. 6102-6113, Dec. 2014.

[3] K. K. Yan and Y. Lu, "Sidelobe reduction in array-pattern synthesis using genetic algorithm," IEEE Trans. Antennas Propag., vol. 45, no. 7, pp. 1117-1122, Jul. 1997.

[4] R. L. Haupt, "Optimized element spacing for low sidelobe concentric ring arrays," IEEE Trans. Antennas Propag., vol. 56, no. 1, pp. 266-268, Jan. 2008.

[5] Y. Chen, S. Yang, and Z. Nie, "Synthesis of uniform amplitude thinned linear phased arrays using the differential evolution algorithm," Electromagnetics, vol. 27, no. 5, pp. 287-297, Jun. 2007.

[6] S. K. Goudos, K. Siakavara, T. Samaras, E. E. Vafiadis, and J. N. Sahalos, "Sparse linear array synthesis with multiple constraints using differential evolution with strategy adaptation," IEEE Antennas Wireless Propag. Lett., vol. 10, pp. 670-673, Jul. 2011.

[7] J. W. Hooker and R. K. Arora, "Optimal thinning levels in linear arrays," IEEE Antennas Wireless Propag. Lett., vol. 9, pp. 771-774, Aug. 2010.

[8] R. Bhattacharya, T. K. Bhattacharyya, and R. Garg, "Position mutated hierarchical particle swarm optimization and its application in synthesis of unequally spaced antenna arrays," IEEE Trans. Antennas Propag., vol. 60, no. 7, pp. 3174-3181, Jul. 2012.

[9] F. Viani, G. Oliveri, and A. Massa, "Compressive sensing pattern matching techniques for synthesizing planar sparse arrays," IEEE Trans. Antennas Propag., vol. 61, no. 9, pp. 4577-4587, Sep. 2013.

[10] X. Zhao, Q. Yang, and Y. Zhang, "Compressed sensing approach for pattern synthesis of maximally sparse non-uniform linear array," IET Microw. Antennas Propag., vol. 8, no. 5, pp. 301-307, Apr. 2014.

[11] G. Oliveri, M. Carlin, and A. Massa, "Complex-weight sparse linear array synthesis by Bayesian compressive sampling," IEEE Trans. Antennas Propag., vol. 60, no. 5, pp. 2309-2326, May 2012.

[12] G. Oliveri and A. Massa, "Bayesian compressive sampling for pattern synthesis with maximally sparse non-uniform linear arrays," IEEE Trans. Antennas Propag., vol. 59, no. 2, pp. 467-481, Feb. 2011.

[13] W. Zhang, L. Li, and F. Li, "Reducing the number of elements in linear and planar antenna arrays with sparseness constrained optimization," IEEE Trans. Antennas Propag., vol. 59, NO. 8, pp. 3106-3111, Aug. 2011. 
[14] Y. Liu, Z. Nie, and Q. H. Liu, "Reducing the number of elements in a linear antenna array by the matrix pencil method," IEEE Trans. Antennas Propag., vol. 56, no. 9, pp. 2955-2962, Sep. 2008.

[15] Y. Liu, Q. H. Liu, and Z. Nie, "Reducing the number of elements in the synthesis of shaped-beam patterns by the forward-backward matrix pencil method," IEEE Trans. Antennas Propag., vol. 58, no. 2, pp. 604-608, Feb. 2010 .

[16] S. E. Nai, W. Ser, Z. L. Yu, and H. Chen, "Beampattern synthesis for linear and planar arrays with antenna selection by convex optimization," IEEE Trans. Antennas Propag., vol. 58, no. 12, pp. 3923-3930, Dec. 2010.

[17] G. Prisco and M. D'Urso, "Maximally sparse arrays via sequential convex optimizations," IEEE Antennas Wireless Propag. Lett., vol. 11, pp. 192-195, Mar. 2012.

[18] B. Fuchs, "Synthesis of sparse arrays with focused or shaped beampattern via sequential convex optimizations," IEEE Trans. Antennas Propag., vol. 60, no. 7, pp. 3499-3503, Jul. 2012.

[19] M. B. Hawes and W. Liu, "Compressive sensing-based approach to the design of linear robust sparse antenna arrays with physical size constraint," IET Microw. Antennas Propag., vol. 8, no. 10, pp. 736-746, Jul. 2014.

[20] A. F. Morabito and P. Rocca, "Reducing the number of elements in phase-only reconfigurable arrays generating sum and difference patterns," IEEE Antennas Wireless Propag. Lett., vol. 14, pp. 1338-1341, Jun. 2015.

[21] A. F. Morabito, A. R. Lagana, G. Sorbello, and T. Isernia, "Maskconstrained power synthesis of maximally sparse linear arrays through a compressive-sensing-driven strategy," J. Electromagn. Waves Appl., vol. 29, no. 10, pp. 1384-1396, 2015.

[22] G. Oliveri, E. T. Bekele, F. Robol, and A. Massa, "Sparsening conformal arrays through a versatile BCS-based method," IEEE Trans. Antennas Propag., vol. 62, no. 4, pp. 1681-1689, Apr. 2014.

[23] J. Gorski, F. Pfeuffer, and K. Klamroth, "Biconvex sets and optimization with biconvex functions: a survey and extensions," Math Method Oper. Res., vol. 66, no. 3, pp. 373-407, Dec. 2007.

[24] F. Wang, V. Balakrishnan, P. Y. Zhou, J. J. Chen, R. Yang, and C. Frank, "Optimal array pattern synthesis using semidefinite programming," IEEE Trans. Signal Process., vol. 51, no. 5, pp. 1172-1183, May 2003. 